\title{
The cerebral correlates of different types of perseveration in the Wisconsin Card Sorting Test
}

\author{
Y Nagahama, T Okina, N Suzuki, H Nabatame, M Matsuda
}

J Neurol Neurosurg Psychiatry 2005;76:169-175. doi: 10.1136/jnnp.2004.039818

See end of article for authors' affiliations

.....................

Correspondence to:

Yasuhiro Nagahama,

Department of Geriatric

Neurology, Shiga Medical

Center, 5-4-30 Moriyama,

Moriyama-city, Shiga

524-8524, Japan;

nagahama@

kuhp.kyoto-u.ac.jp

Received 20February 2004

In revised form

25 April 2004

Accepted 6 May 2004

\begin{abstract}
Objectives: To explore the neural substrates corresponding to the perseverative errors in the Wisconsin Card Sorting Test (WCST).

Methods: The study examined the correlations between the WCST performances and the SPECT measurements of regional cerebral blood flow (rCBF) in subjects with neurodegenerative dementia. Negative non-linear correlations between the rCBF and the two different types of the perseverative errors ("stuck-in-set" and "recurrent" perseverative errors) were calculated on a voxel basis and volume-ofinterest basis in the mixed groups of 72 elderly and dementia patients.

Results: The stuck-in-set perseverative error was associated with the reduced rCBF in the rostrodorsal prefrontal cortex, whereas the recurrent perseverative error was related to the left parietal activity but not to the prefrontal activity.

Conclusions: These findings augment evidence that the rostrodorsal prefrontal cortex crucially mediates attentional set shifting, and suggest that the stuck-in-set perseverative errors would be a true pathognomonic sign of frontal dysfunction. Moreover, this study shows that the recurrent perseverative errors may not be associated closely with the prefrontal function, suggesting that this error and the stuckin-set error should be differentially estimated in the WCST.
\end{abstract}

$\mathrm{T}$ he Wisconsin Card Sorting Test (WCST) has been widely used as a means of assessing cognitive flexibility-that is, the ability to shift cognitive set from one perceptual attribute of a complex visual stimulus to another ("attentional set shifting"). Typically, patients with frontal lobe damage continued to sort the cards according to a previous rule, even after the rule has clearly been changed. ${ }^{1}$ This failure is known as "perseveration". Therefore, poor performance on the WCST, especially an increase in the number of perseverative errors, has been associated with frontal lobe dysfunction. ${ }^{12}$ Although there are some controversial findings, ${ }^{3}{ }^{4}$ cumulative evidence suggests that the WCST is sensitive to frontal brain damage. ${ }^{125-7}$ However, as the WCST is a complex problem solving task that requires multiple cognitive processes rather than a single unitary function, ${ }^{89}$ it is likely that not all underlying cognitive processes contributing to the WCST performance are because of the functions of the frontal lobe. Although the study of patients with well-restricted focal frontal and non-frontal lesions may be essential to dissociate such processes, only a small number of studies have evaluated this. ${ }^{78}$

Functional neuroimaging studies have confirmed the involvement of the prefrontal cortex (PFC) in the performance of the WCST. ${ }^{10-13}$ There are some evidence that distinct prefrontal regions may process different cognitive information during the WCST. A recent functional magnetic resonance imaging (fMRI) study ${ }^{14}$ revealed that the rostrodorsal PFC and posterior PFC showed dissociable neural activations in the two different levels of response selection-that of "attentional set shift" and that of "reversal shift". This suggests that the reorganisation of the stimulus response associations (reversal shift) is associated with the posterior PFC, whereas the higher order shifting of attention (attentional set shift) is mediated by the rostrodorsal PFC. Monchi et al ${ }^{12}$ also revealed that the mid-dorsolateral PFC increased activity while subjects received either positive or negative feedbackthat is, at the point when the subjects must monitor and update the information in working memory. In contrast, the posterior PFC response was less specific and increase in activity occurred during both the reception of feedback and the response period. These findings suggest that the rostrodorsal PFC and the posterior PFC have different neurophysiological roles in the process of shifting behaviour. However, neuroimaging in healthy subjects can, in principle, identify the set of regions that are sufficient for the cognitive operation but does not distinguish whether the identified regions are undoubtedly necessary to perform the task or not. ${ }^{15}$ To establish the requirement of such component brain areas for the task, it is necessary to examine whether the dysfunction in the regions is associated with a deficit in the specified function.

To analyse the neuropathological correlates of the perseverative behaviour it is important to recognise that perseveration is not a unitary phenomenon but a collection of different forms of behaviour. Previous researches demonstrated that perseveration consists of three types: ${ }^{16}$ (1) inappropriate repetition of a response without interruption, (2) recurrence of a previous response with interruption by intervening responses, and (3) inappropriate maintenance of a category or framework of activity. Several neuropsychological studies suggested that the perseverative errors in the WCST scored by the traditional system could be classified into at least two different types of perseverative responses with or without interruption by the intervening responses. ${ }^{77^{18}}$ It is expected that these different types of perseverative errors are associated with distinctive neuroanatomical systems.

Abbreviations: AD, Alzheimer's disease; BA, Brodmann's area; $C T$, computed tomography; $f M R I$, functional magnetic resonance imaging; FTD, frontotemporal lobar degeneration; FWHM, full width at half maximum; HMPAO, hexamethyl-propyleneamine oxime; $\mathrm{MCl}$, mild cognitive impairment; MMSE, Mini-Mental State Examination; PFC, prefrontal cortex; Rec-PE, recurrent perseverative error; $r C B F$, regional cerebral blood flow; SPECT, single photon emission computed tomography; SPM, Statistical Parametric Mapping; SiS-PE, stuck-in-set perseverative error; WCST, Wisconsin Card Sorting Test; VOI, volume of interests 
To explore the neural substrates corresponding to the particular perseverative errors in the WCST, we planned to examine the correlations between the WCST performances and the resting regional cerebral blood flow ( $\mathrm{rCBF}$ ) in patients with neurodegenerative dementia. Previous neuroimaging studies have shown that analysis of the correlations between cognitive performance and resting cerebral metabolism and blood flow is sensitive to unravel the neural substrates of cognitive impairments. ${ }^{19-21}$ Most previous studies that examined the relation between the behavioural parameters and the neural activity employed a simple linear function as the regressor. However, the linear function may be not good enough to characterise brain regions by their rCBF value in relation to the parameters. There is now considerable evidence to suggest that ageing and disease processes in the brain introduce compensatory mechanisms to resist the "to be reduced" cognitive performances. Recent neuroimaging studies demonstrated that in elderly people and early Alzheimer's disease (AD) patients the neural activity in several brain regions, such as prefrontal and hippocampal cortex, shows greater increase than that in young patients during cognitive tasks, despite the patients' task performance being poorer than the healthy subjects. ${ }^{22-25}$ Furthermore, a histochemical study showed that the choline acetyltransferase activity in the frontal and hippocampal cortex was up-regulated in subjects with mild cognitive impairment (MCI) and these activities were actually reduced in the clinically evident $\mathrm{AD} .^{26}$ These physiological and histochemical findings suggest that during early cognitive decline neural activity in these brain regions could be reserved to compensate for degenerative processes and may protect these individuals from becoming obviously demented. We can therefore hypothesise that in the parametric regression analysis the relation between the $\mathrm{rCBF}$ and the reduced task performances might be non-linear; during the performance reduction is mild, the neural activity-that is, $\mathrm{rCBF}$-in the corresponding brain regions is preserved because of a compensatory mechanism and then the rCBF is decreased as the task performance is further impaired.

In general, how good the regression fits depends on the type of the basis function chosen. As the relation between parameters and rCBF could be non-linear, the a priori definition of a fit function as a linear one might lead to an insufficient or partial result. Thus, we modelled the rCBF changes with a non-linear function-for example, a second order polynomial. We expected that the use of the non-linear basis function in conjunction with the general linear model facilitates the detection of $\mathrm{rCBF}$ changes in brain regions that might not have been so evident using simple linear regression.

\section{SUBJECTS}

Seventy two patients were included in the study: six elderly subjects (54-86 years), 12 patients with MCI (60-79 years), three patients with frontotemporal lobar degeneration (FTD, 60-76 years), and 51 patients with probable AD (66-85 years). All were outpatients who attended the Memory Clinic, Shiga Medical Center between July 2000 and March 2003.

Patients with AD fulfilled the DSM III-R criteria for "primary degenerative dementia" 27 and NINCDS/ADRDA criteria for probable AD. ${ }^{28}$ Patients with amnestic MCI were diagnosed according to recommendation criteria of the American Academy of Neurology. ${ }^{29}$ Patients with frontotemporal lobar degeneration were diagnosed using the clinical diagnostic criteria for FTD. ${ }^{30}$

All patients were interviewed and received a comprehensive neurological examination prior to inclusion in the study. Exclusion criteria included a history of stroke, psychiatric disorder, significant head trauma, alcohol abuse, or evidence of other systemic or neurological disorders that may compromise cognition. All patients received the Mini-Mental State Examination (MMSE) $)^{31}$ and severely demented patients (whose MMSE score was less than 13) were excluded from the study. All patients were scanned with head computed tomography (CT) or MRI, and no abnormalities were found other than atrophy and/or mild periventricular low densities. Most patients received no psychotropic medication in the month preceding the study except for 11 patients: seven $\mathrm{AD}$ were medicated with donepezil, two $\mathrm{AD}$ with donepezil and tiapride, one $\mathrm{AD}$ with paroxetine, one FTD with maprotiline. All the neuropsychological tests and the neuroimaging were parts of routine clinical examinations, and informed consent was obtained from all patients and/or caregivers concerning the nature and purpose of the procedures.

\section{The WCST}

A computerised, modified version of the WCST was administered to all the patients. In the WCST, the patients were instructed to match a "response" card to one of the four "stimulus" cards on the basis of one of the three possible categories of number, colour, or shape, by clicking one of the stimulus cards using a mouse. In some patients who were unfamiliar with using a computer mouse, the patients selected their answers by pointing at one of the stimulus cards with their index finger and the examiner clicked them. As in the Nelson's version, ${ }^{2}$ we used the $48(2 \times 24)$ response cards that share one and only one attribute with the stimulus cards. Patients were not informed of the correct sorting principle, nor were they told when the principle would shift during the test, but they were informed of the three possible categories before testing. The patients were required to determine which one was correct based solely on the feedback that indicated whether each response was "correct" or "wrong". When the patient maintained a correct progression through six trials, the rule was changed without warning and they had to shift the sorting rule from the previously relevant category to the other one to yield correct answers. Unlike the common forms of the traditional WCST in which the test ends after six correct categories were achieved, the testing in this study continued until all 48 cards were sorted.

The responses were scored according to Heaton's criteria ${ }^{32}$ but with one minor exception: the first unambiguous error repeating the previously correct principle was not scored as perseveration because the subject could not yet have received feedback indicating that the previously correct principle was now incorrect. ${ }^{33}$ We first calculated the raw scores of Heaton's perseverative errors and non-perseverative errors. Heaton's perseverative errors include the following three criteria:

- Incorrect responses that have been correct in the previous stage.

- In cases of patients who were so perseverative that they never attained the first category, the first incorrect unambiguous response in stage one was regarded as the "perseverated to" criterion.

- If the subject made three unambiguous incorrect responses in succession according to another principlethat is, the principle that was neither the correct one in the current stage nor the one that was defined as the perseverated to principle according to the rules given above-the perseverated to criterion was changed to that principle.

Then we divided the perseverative errors into the two different perseverative scores: the "stuck-in-set" perseverative error (SiS-PE) and the "recurrent" perseverative error (Rec-PE). The SiS-PE is similar to the perseverative errors 
defined by Nelson: exact repetitions of the immediately preceding incorrect response. It reflects a "short range" perseveration in which the patients could not shift their responding even when they knew the rule was wrong-that is, the failure to shift attentional set. Rec-PE is the inappropriate repetition of a previous response after an intervening response, in which the patients could change their sorting principle once but recurred to the irrelevant one (see table 1 for an example).

A total of $57.5 \%$ of the perseverative errors in this study were SiS-PE and $42.5 \%$ of the perseverative errors were RecPE. The correlation between the SiS-PE and Rec-PE was not significant $(\mathrm{r}=0.25)$. Thus, these two perseverative scores seem to reflect different aspects of perseverative behaviour as previously mentioned. ${ }^{7}{ }^{17} 18$ We finally therefore used the scores of the SiS-PE and Rec-PE for the subsequent image analyses.

\section{SPECT scanning}

Within a month of the neuropsychological evaluations, the resting $\mathrm{rCBF}$ in all the subjects was evaluated using single photon emission computed tomography (SPECT). Subjects lay on the scanner bed with their eyes closed and $740 \mathrm{MBq}$ of [ ${ }^{99 \mathrm{~m}} \mathrm{Tc}$ ]hexamethyl-propyleneamine oxime (HMPAO) was administered intravenously. After $15 \mathrm{~min}$ of injection, the SPECT image was acquired over a period of 20 min using a triple-head SPECT scanner (GCA-9300A/UI; Toshiba Medical, Tokyo, Japan) with high-resolution fan-beam collimators. The axial field of view of the cameras was $22 \mathrm{~cm}$ and the resolution of the system was $7.5 \mathrm{~mm}$ full width at half maximum (FWHM) in the centre of view. Projection data, collected in a $128 \times 128$ matrix, were prefiltered with a Butterworth filter with order 8 and a cut off frequency of 0.11 cycles/pixel. Transaxial images were reconstructed with a Ramp back projection filter. The post-reconstruction attenuation correction was not applied. The reconstruction yielded $1.7 \times 1.7 \times 1.7 \mathrm{~mm}$ voxels with a $128 \times 128$ matrix and 80 slices.

\section{Image processing}

The SPECT data were processed with Statistical Parametric Mapping (SPM2, Wellcome Department of Cognitive Neurology, London, UK). All the SPECT images were coregistered and transformed into a standard stereotactic anatomical space (International Consortium of Brain Mapping space $)^{34}$ using the original disease-specific SPECT template (made from the SPECT images of $33 \mathrm{AD}$ and nine MCI patients). The resulting voxel size was $2 \times 2 \times 2 \mathrm{~mm}$. The images then were smoothed with a $10 \mathrm{~mm}$ gaussian filter to reduce the variance due to individual anatomical variability. Thereafter, the effect of differences in global CBF across subjects was removed using proportional scaling. This process equalised the mean CBF value to $50 \mathrm{ml} / \mathrm{dl} / \mathrm{min}$ across subjects, and generated an associated adjusted error variance

Table 1 Examples of responses scored if colour was the correct sorting principle and figure the previous one

\begin{tabular}{ll}
\hline Responses & Scores \\
\hline C & Correct \\
$F$ & Rec-PE \\
F & SiS-PE \\
$N$ & Non-perseverative error \\
F & Rec-PE \\
$N$ & Non-perseverative error \\
$N$ & SiS-PE \\
$N$ & SiS-PE \\
\hline
\end{tabular}

$C$, colour; $F$, figure; $N$, number; Rec-PE, recurrent perseverative error; $\mathrm{SiS}-\mathrm{PE}$, stuck-in-set perseverative error. for each voxel; therefore this allowed us to make comparisons of the mean $\mathrm{CBF}$ distributions across all subjects.

Although the regional cerebral metabolism and rCBF could be affected by neuronal lesions - that is, cerebral atrophy—as well as synaptic dysfunction, all studies that performed voxel-based atrophy correction of glucose metabolism have concluded that the reduced cerebral metabolism represents a true loss of functional activity and not simply an artefact caused by brain atrophy. ${ }^{35}$ Moreover, some correlational studies (not voxel based) took brain atrophy into account in their analysis and the results were nearly the same as those obtained without correction for atrophy; ${ }^{37}$ we therefore did not perform atrophy correction in the subsequent analyses.

\section{Voxel by voxel parametric analysis}

The parametric approach models changes in rCBF as a function of the experimental parameter by using regression analysis. In general, SPM uses the general linear model to build $F$ - or $t$-statistic fields-that is, $\operatorname{SPM}\{\mathrm{F}\}$ or $\operatorname{SPM}\{\mathrm{t}\} .^{38}$ In the special case of parametric studies it is used to make statistical inferences about the correlation of rCBF and a study parameter. In the present study, we modelled changes in the rCBF with a non-linear function of the WCST performance parameters using a regression with a secondorder polynomial function. As we expected that rCBF in the patient's brain will decrease monotonically in relation to the increase of their WCST errors, voxel-specific $t$-statistics were used to make inferences about the negative nonlinear regression of $\mathrm{rCBF}$ on the study parameters. These analyses generated statistical parametric maps of the $t$ statistic (SPM $\{\mathrm{t}\}$ ), which were subsequently converted to unit normal distribution ( $\operatorname{SPM}\{Z\})$. Age and the MMSE scores were treated as covariates of no interest, so that the confounding effects of age and dementia severity on the rCBF distribution were covaried out. In this analysis, the statistical threshold was set at $\mathrm{p}<0.001 \quad(\mathrm{Z}>3.09)$ without correction for multiple comparisons. This threshold is regarded as sufficiently conservative to protect against false-positive results in PET analysis. ${ }^{39}$

The stereotactic coordinates of Talairach and Tournoux ${ }^{40}$ were used to report the brain areas, but descriptions of the anatomical location were also based on visual inspection of the standard structural MRI.

\section{Volumes of interests correlation analysis}

To clarify which of the prefrontal areas was actually associated with the WCST performances, we evaluated correlations between the rCBF in several predetermined prefronal regions and the first and second order polynomial expansion of the WCST error scores. MRIcro software (Rorden C, http://www.mricro.com) was used for the volume of interests (VOI) analyses. Four different prefrontal areas that can be associated with cognitive processes in the WCST were determined based on the previous neuroimaging studies: (1) rostrodorsal PFC $(x= \pm 34, y=50, z=14)$, which is an anterior part of the middle frontal gyrus near the frontal pole, and regarded as BA 10 or most rostral part of BA 46; (2) posterior PFC $(x= \pm 46, y=8, z=28)$, which was situated in the inferior frontal sulcus near the junction with the precentral sulcus and corresponded to BA 8A/44 or 45 ; (3) ventrolateral PFC $(x= \pm 36, y=28, z=-2)$, which is located around the oppercular part of the inferior frontal gyrus, and regarded as Brodmann's area (BA) 47/12; and (4) anterior cingulate area $(x= \pm 8, y=20, z=46)$. The activation peaks in the previous fMRI and PET studies related to the WCST were selected ${ }^{11-1441}$ and averaged to yield the representative stereotactic coordinates for each prefrontal region. Then, the coordinates were slightly modified to be positioned on the cortical CBF of the SPECT images in the present study. 
Spherical VOIs $(20 \mathrm{~mm}$ in diameter) centred on the predetermined prefrontal regions were drawn on all $10 \mathrm{~mm}$ smoothed, anatomically and globally normalised SPECT images, and every voxel value in the VOI was averaged to calculate the rCBF. To determine the relation between the WCST parameters and the $\mathrm{rCBF}$, we evaluated partial correlation coefficients between the WCST performances and the rCBF, including age and the MMSE scores as confounding parameters. For the partial correlation coefficients, statistical significance level was set at $\mathrm{p}<0.01$.

\section{RESULTS}

The SPM analyses demonstrated that the SiS-PE scores showed significant non-linear regression with the $\mathrm{rCBF}$ in the right rostrodorsal PFC (rostral part of the middle frontal gyrus, the coordinates, $\mathrm{Z}$ score, and the cluster size $\{\mathrm{k}\}$ were $\mathrm{x}=32, \mathrm{y}=48, \mathrm{z}=10, \mathrm{Z}=3.36, \mathrm{k}=118$ voxels), left rostrodorsal PFC (the middle frontal gyrus, $x=-32, y=58, z=20$, $\mathrm{Z}=3.29, \mathrm{k}=11$ voxels), and the left frontopolar cortex (the frontomarginal gyrus, $x=-26, y=52, z=-6, z=3.43$, $\mathrm{k}=39$ voxels)(fig 1 ). In contrast, the Rec-PE showed significant regression with the $\mathrm{rCBF}$ in the left inferior parietal cortex (around the angular gyrus, $x=-38, y=-56$, $\mathrm{Z}=40, \mathrm{Z}=3.27, \mathrm{k}=35$ voxels)(fig 2 ). Even when the statistical threshold was lowered to $\mathrm{p}<0.01$, the Rec-PE was not associated with the $\mathrm{rCBF}$ in the prescribed frontal cortices, whereas the SiS-PE was only related to the reduced $\mathrm{rCBF}$ in these prefrontal regions. These results were unchanged when the three FTD patients were excluded from the analyses.

The VOI analyses also demonstrated that the rCBF in the right rostrodorsal PFC showed significant non-linear correlation with the SiS-PE score $(\mathrm{r}=-0.355, \mathrm{p}=0.0025)$. The nonlinear correlation between the $\mathrm{rCBF}$ in the left rostrodorsal
PFC and the SiS-PE score approached to the significance level $(\mathrm{r}=-0.293, \mathrm{p}=0.013)$. The linear correlation between the rCBF in the right rostrodorsal PFC and the SiS-PE score also approached to the significance level $(\mathrm{r}=-0.30, \mathrm{p}=0.011)$. The rCBF in the other prefrontal areas did not show any significant correlations with the SiS-PE score. The Rec-PE did not show any significant correlations with the rCBF in the prefronal areas in the VOI analyses.

\section{DISCUSSIONS}

We determined the sites of significant correlations between two different types of the WCST perseverative errors and rCBF in the group of degenerative dementia. First, the number of SiS-PE score was significantly associated with the reduced $\mathrm{rCBF}$ in the rostrodorsal PFC, supporting the conclusion in our previous fMRI study in healthy subjects. ${ }^{14}$ Second, the Rec-PE was correlated to the left parietal activity but not to the prefrontal activity, suggesting its differential psychophysiological nature from the SiS-PE.

We pooled SPECT data from four different clinical entities of AD, MCI, FTD, and healthy elderly for the present analyses. This involvement of the heterogeneous groups enabled us to extract the brain regions that are associated with the WCST impairments, independently of clinical diagnosis. The essence of this approach is that the basic units of classification in psychopathology are neither diseases nor syndromes but psychological dysfunctions, and that shared behavioural deficits in nosologically distinct neuropsychiatric disorders may have common neurophysiological correlates. ${ }^{42}$ Within this framework, the previous study showed that poverty of speech is associated with decreased rCBF in the left dorsolateral PFC in depression and schizophrenia in a manner that is independent of diagnosis. ${ }^{43}$

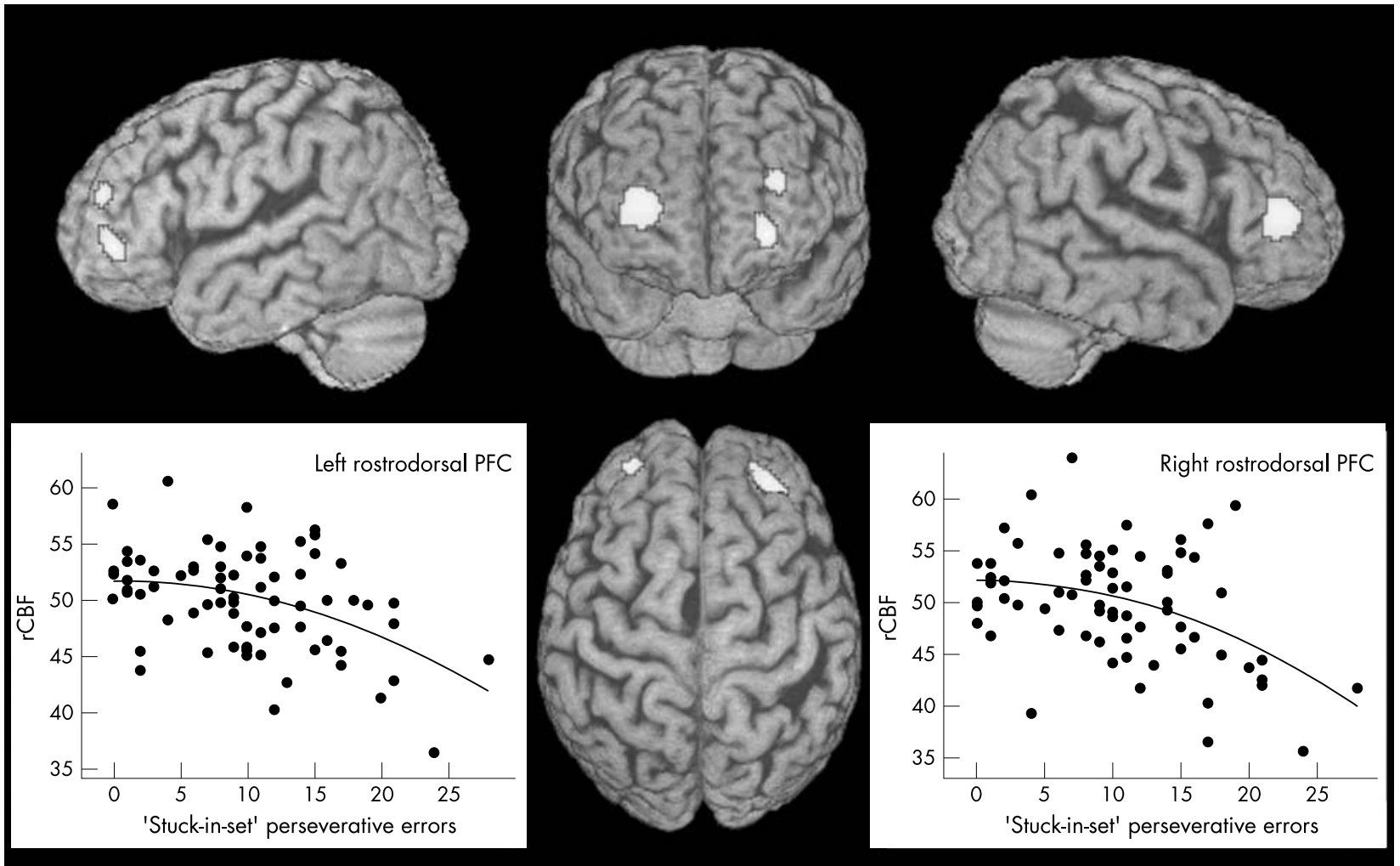

Figure 1 The rostrodorsal prefrontal cortex showing significant association with the number of the stuck-in-set perseverative errors in the Wisconsin Card Sorting Test. The SPM $\{Z\}$ result image was overlaid on the normalised T1 image from a subject with mild Alzheimer's disease. PFC, prefrontal cortex; rCBF, regional cerebral blood flow ( $\mathrm{ml} / \mathrm{dl} / \mathrm{min})$. 


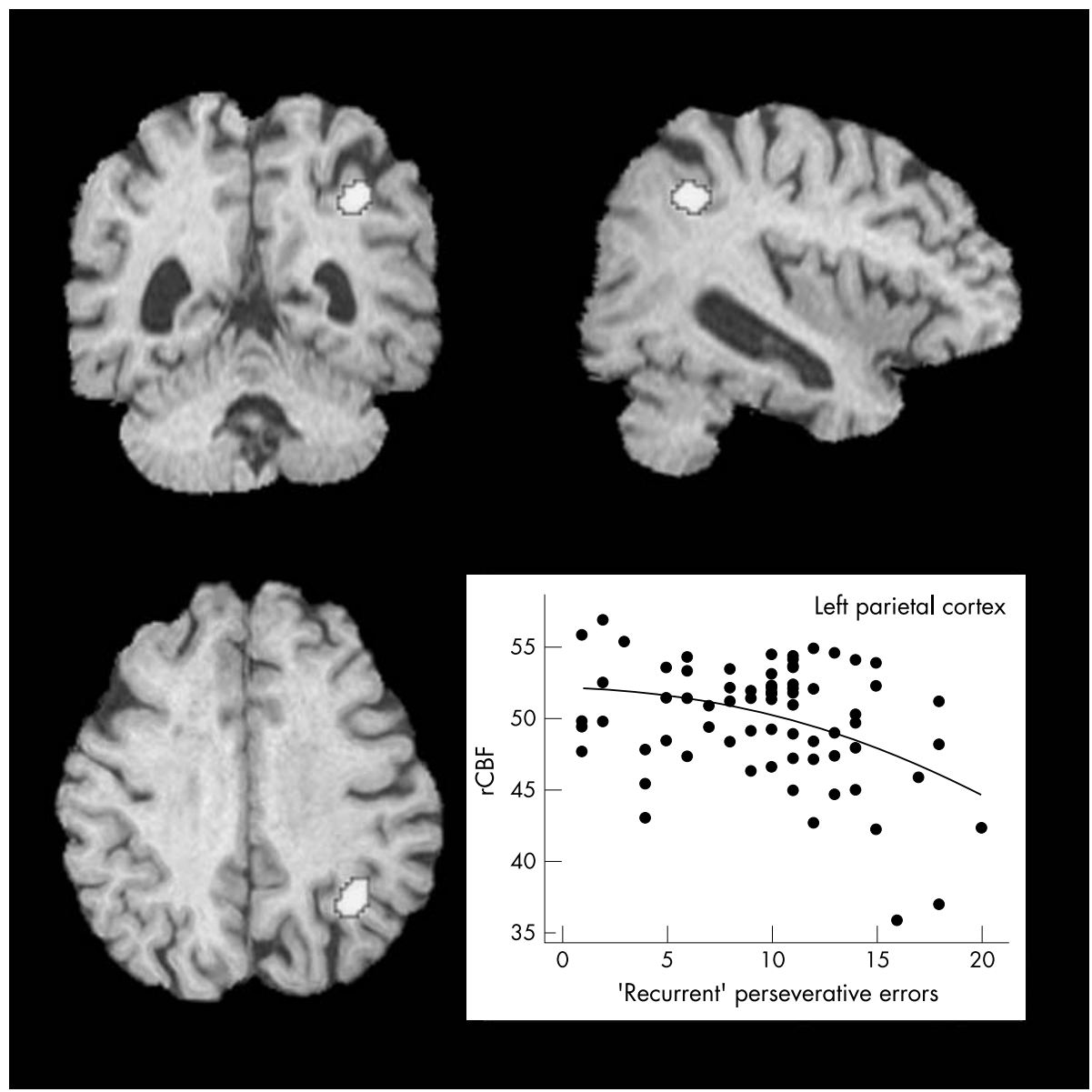

Figure 2 The left inferior parietal cortex showing significant association with the number of the recurrent perseverative errors in the Wisconsin Card Sorting Test. $\mathrm{rCBF}$, regional cerebral blood flow $(\mathrm{ml} / \mathrm{dl} / \mathrm{min})$.

The correlation of rCBF decrease with the impaired WCST performances in the present study could be confounded by disease severity and ageing effect. Many neuroimaging studies have shown that the distribution of the rCBF decrease depends on the stage of dementia. In moderate to severely demented $\mathrm{AD}$ patients, frontal hypometabolism is as severe as that in the parietotemporal area. ${ }^{44}{ }^{45}$ Ageing is also associated with the rCBF decline in the frontotemporal cortex. ${ }^{46}{ }^{47}$ Therefore, one could argue that the rCBF decrease in the several brain regions, such as the rostrodorsal PFC, may be because of progression in dementia itself and does not truly relate to neuropsychological performances. However, this is unlikely because of the following reasons. First, in $\mathrm{AD}$ (a large number of patients in this study) the most consistent finding across the neuroimaging research was that the gradual rCBF decline according to the disease progression was observed in the parietotemporal association cortex..$^{44} 48$ If the rCBF decrease is associated with progression of dementia, it should be detected in the posterior association cortices. However, the reduction of the rCBF in relation to the SiS-PE was observed only in the prefrontal cortex. Second, the WCST performances in our sample showed no significant correlation with dementia severity-that is, MMSE score-or age (data not shown). In addition, the influence of age and dementia severity was controlled by setting age and the MMSE score as confounding variables in all the regression analyses.

\section{Differential involvement of the prefrontal regions}

A principal aim of this study was to investigate the neural correlates of two distinct kinds of perseverative errors implicit in the WCST. The results of the present study unequivocally demonstrate that the SiS-PE score is particularly associated with the rCBF reduction in the bilateral rostrodorsal PFC (right $>$ left) and the left frontopolar cortex. As the SiS-PE reflects failure of shifting attentional set away from a previously reinforced stimulus dimension towards a previously irrelevant stimulus dimension, these findings augment evidence that attentional set shifting is crucially mediated by predominantly the rostrodorsal PFC. Stuss et al ${ }^{7}$ reported that the right frontal patients had significantly elevated "perseveration to the preceding response" score in the WCST, which was defined similar to the SiS-PE in this study, whereas very few non-frontal patients exhibited these errors. Our previous study in normal subjects revealed that the right rostrodorsal PFC was activated only at the time of attentional set shifting and that it may be a critical area in shifting an attentional set between different perceptual dimensions. ${ }^{14}$ The present results complement and strongly support our previous findings, and suggest that the SiS-PE would be a true pathognomonic sign of frontal dysfunction.

Previous findings in the other imaging studies are also consistent with this notion. PET studies in normal subjects have shown that the rostrodorsal PFC was consistently activated during the performance of the WCST. ${ }^{101141}$ In the event-related fMRI experiment, Konishi et al ${ }^{13}$ showed that transient neural activity was observed in the left frontal cortex, including the rostrodorsal PFC at the time of updating cognitive set during the WCST. Further support for a contribution of this brain region on the perceptual 
dimensional change comes from a recent study that compared cross-dimensional changes with within-dimensional changes in a sophisticated set shifting task analogous to the WCST. ${ }^{49}$ The comparison of extradimensional and intradimensional shifts revealed a right dorsolateral prefrontal activation (BA 9/46), which was very close to our rostrodorsal PFC activation, suggesting that control of attentional set is crucially mediated by this prefrontal region.

In addition to the rostrodorsal PFC, the rCBF reduction in the left frontopolar cortex was also associated with the increased SiS-PE. The possible involvement of the left frontopolar cortex during the cross-dimensional shifts is supported by several previous studies. Previous PET studies demonstrated that the left frontopolar activation was observed during the performance of the WCST. ${ }^{101141}$ A recent fMRI study of visual singleton (odd-one-out) feature search found that the left frontopolar cortex appears to be exclusively involved in the process of attentional switching from one visual dimension to another. ${ }^{50}$

Previous fMRI studies of the WCST showed that the neural activity in the posterior PFC and the ventrolateral PFC also increased during set shifting trials. ${ }^{12}{ }^{51}$ However, both SPM and VOI analyses in the present study failed to show any significant correlation between the rCBF in these regions and the perseverative error scores in the WCST, suggesting these prefrontal subregions other than the rostrodorsal PFC may play different roles than attentional set shifting. Cumulative evidence suggests that the posterior PFC is less specific to the attentional switching and may be involved in the selection of the appropriate response based on the currently relevant rule. ${ }^{12}{ }^{14}$ The ventrolateral PFC region is commonly activated if it is necessary to plan a response to the negative feedback during the type of go/nogo tasks or reversal learning task. ${ }^{52}$ In the monkey, lesions of the ventrolateral prefrontal convexity impaired the spatial and non-spatial reversal learning. ${ }^{54-56}$ Furthermore, a recent study by Cools et al ${ }^{57}$ showed that the ventrolateral PFC is primarily associated with reversal learning and is not explained by negative feedback. Thus, the ventrolaterel PFC may be involved in shifting of lower level stimulus reward associations-that is, reversal learning-whereas higher level cognitive set shifting is mediated by the rostrodorsal PFC, which was the only prefrontal subregion showing a significant correlation with the SiS-PE scores in the WCST. This difference in the dorsolateral versus ventrolateral PFC involvement in the WCST is consistent with a theory that emphasises the difference in the role of the mid-dorsolateral prefrontal area, which is necessary for the monitoring of information in working memory, as opposed to the midventrolateral prefrontal area, which is involved in more basic executive processes, such as the active comparison of stimuli held in working memory. ${ }^{58} 59$

\section{Different types of perseverative errors}

Another important finding in the present study was that the Rec-PE was differentially associated with the rCBF reduction in the left inferior parietal cortex and showed no significant correlation with the rCBF in the prefrontal region. Although we cannot exclude that this correlation is false positive, involvement of the parietal cortex was reported in previous PET and fMRI studies of the WCST. ${ }^{10-12}$ Sandson and Albert ${ }^{60}$ suggested, based on their study of brain damaged patients, that the recurrent perseveration is linked to left temporalparietal damage and not to frontal damage. Several studies in healthy subjects showed that the parietal cortex is involved in processing of visual attention. Fink et $a l^{61}$ revealed that object based and space based attention share common neural mechanisms in the left lateral inferior and bilateral medial superior parietal areas. Wojciulik et al ${ }^{62}$ examined brain activation during two different spatial attention tasks and a non-spatial attention (conjunction and a feature) task to determine whether different kinds of visual attention rely on a common neural substrate. All spatial and non-spatial attention tasks activated the inferior parietal lobules (the junction of intraparietal and transverse occipital sulci and the anterior intraparietal sulcus) bilaterally. They emphasised that these parietal regions play a more general role in visual attention, and suggested that the parietal cortex may perform an inhibitory function in selective attention suppressing task irrelevant distractors. This theoretical position may be consistent with the results in the present study because the RecPE in the WCST may be closely related to failure to suppress the previously relevant but currently irrelevant visual dimension. Although the relation between the type of perseverative errors on the WCST and the localised parietal lesion has seldom been examined, impairment on the WCST was reported in one patient with left parietal damage (patient TW1124). ${ }^{8}$ The present results suggest that the SiS-PE and Rec-PE may be differentiated not only behaviourally but also on their neurophysiological basis. These two types of perseveration should be separately estimated in future studies of the WCST in brain lesioned patients.

\section{Right versus left prefrontal cortex in the WCST}

The results of the present study and our previous fMRI study ${ }^{14}$ suggest right frontal predominance in the process of the attentional set shifting. The right dominant tendency in the WCST has emerged in several studies, ${ }^{25}{ }^{5}$ although some studies found the opposite hemispheric effect. ${ }^{3}$ Stuss et al ${ }^{7}$ recently demonstrated that patients with either left or right focal prefrontal lesion were impaired on the "perseveration to the preceding response" score in the WCST, but the right prefrontal group was impaired more severely than the left prefrontal group.

It is possible that the discrepancy of the hemispheric effect among previous studies is associated with verbal mediation during the performance of the WCST. Several neuroimaging studies suggested that when abstract-that is, difficult to verbalise- figures or colours are used in the tasks and covert verbalisation is effectively prohibited, the right dorsolateral PFC is differentially activated in relation to the attentional set shifting. ${ }^{14963}$ In contrast, the bilateral PFC is active during the performance of the original WCST. ${ }^{10}{ }^{11}$ These findings suggest that although the essential process of the visual dimensional changes is mediated by the non-verbal systems in the right PFC, the set shifting in the WCST could be also performed through the verbal systems in the left hemisphere.

Overall, the present data provide, along with the previous results of activation study in healthy subjects, converging evidence concerning the close relationship between the failure in shifting attentional set and the dysfunction in the rostrodorsal PFC. Furthermore, this study showed that the Rec-PE may not be associated closely with the function of the prefrontal cortex, suggesting that the SiS-PE and Rec-PE should be differentially estimated in the WCST. From these neuropsychological standpoints, future re-examination of the WCST performances in the focal brain damaged patients would be of considerable interest.
Authors' affiliations
Y Nagahama, T Okina, N Suzuki, M Matsuda, Department of Geriatric Neurology, Shiga Medical Center, 5-4-30 Moriyama, Moriyama-city, Shiga 524-8524, Japan
H Nabatame, Department of Neurology, Shiga Medical Center
Competing interests: none declared. 


\section{REFERENCES}

1 Milner B. Effects of different brain lesions on card sorting. Arch Neurol 1963:9:90-100.

2 Nelson HE. A modified card sorting test sensitive to frontal lobe defects. Cortex 1976;12:313-24.

3 Grafman J, Jonas B, Salazar A. Wisconsin Card Sorting Test performance based on location and size of neuroanatomical lesion in Vietnam veterans with penetrating head injury. Percept Mot Skills 1990;71:1120-22.

4 van den Broek MD, Bradshaw CM, Szabadi E. Utility of the Modified Wisconsin Card Sorting Test in neuropsychological assessment. Br J Clin Psychol 1993;32:333-43.

5 Drewe EA. The effect of type and area of brain lesion on Wisconsin card sorting test performance. Cortex 1974;10:159-70.

6 Robinson AL, Heaton RK, Lehman RAW, et al. The utility of the Wisconsin Card Sorting Test in detecting and localizing frontal lobe lesions. J Consult Clin Psychol 1980;48:605-14.

7 Stuss DT, Levine B, Alexander MP, et al. Wisconsin Card Sorting Test performance in patients with focal frontal and posterior brain damage: effects of lesion location and test structure on separable cognitive processes. Neuropsychologia 2000;38:388-402

8 Anderson SW, Damasio H, Jones RD, et al. Wisconsin Card Sorting Test performance as a measure of frontal lobe damage. J Clin Exp Neuropsychol 1991;13:909-22.

9 Dehaene S, Changeux JP. The Wisconsin Card Sorting Test: theoretical analysis and modeling in a neuronal network. Cereb Cortex 1991;1:62-79.

10 Berman KF, Ostrem JL, Randolph C, et al. Physiological activation of a cortical network during performance of the Wisconsin Card Sorting Test: a positron emission tomography study. Neuropsychologia 1995;33:1027-46.

11 Nagahama Y, Fukuyama H, Yamauchi H, et al. Cerebral activation during performance of a Card Sorting Test. Brain 1996;1 19:1667-75.

12 Monchi O, Petrides M, Petre V, et al. Wisconsin Card Sorting revisited: distinct neural circuits participating in different stages of the task identified by eventrelated functional magnetic resonance imaging. I Neurosci 2001;21:7733-41.

13 Konishi S, Hayashi T, Uchida I, et al. Hemispheric asymmetry in human lateral prefrontal cortex during cognitive set shiffing. Proc Natl Acad Sci USA 2002;99:7803-8.

14 Nagahama Y, Okada T, Katsumi Y, et al. Dissociable mechanisms of attentional control within the human prefrontal cortex. Cereb Cortex 2001;11:85-92.

15 Price CJ, Mummery CJ, Moore CJ, et al. Delineating necessary and sufficient neural systems with functional imaging studies of neuropsychological patients. $J$ Cogn Neurosci 1999;11:371-82.

16 Hotz G, Helm-Estabrooks N. Perseveration. Part I: a review. Brain Inj 1995:9:151-9.

17 Nagahama Y, Okina T, Suzuki N, et al. Factor structure of a modified version of the wisconsin card sorting test: an analysis of executive deficit in Alzheimer's disease and mild cognitive impairment. Dement Geriatr Cogn Disord 2003;16:103-12.

18 Parker D, Crawford J. Assessment of frontal lobe dysfunction. In: Crawford J, Parker D, McKinlay W, eds. A handbook of neuropsychological assessment. Hove, UK: Lawrence Erlbaum, 1992:267-91.

19 Perani D, Bressi S, Cappa SF, et al. Evidence of multiple memory systems in the human brain. A [18F]FDG PET metabolic study. Brain 1993;1 16:903-19.

20 Mega MS, Lee L, Dinov ID, et al. Cerebral correlates of psychotic symptoms in Alzheimer's disease. J Neurol Neurosurg Psychiatry 2000;69:167-71.

21 Desgranges B, Baron JC, de la Sayette V, et al. The neural substrates of memory systems impairment in Alzheimer's disease. A PET study of resting brain glucose utilization. Brain 1998;121:611-31.

22 Backman L, Almkvist O, Andersson J, et al. Brain activation in young and older adults during implicit and explicit retrieval. J Cogn Neurosci 1997:9:378-91.

23 Backman L, Andersson JL, Nyberg L, et al. Brain regions associated with episodic retrieval in normal aging and Alzheimer's disease. Neurology 1999;52:1861-70.

24 Bookheimer SY, Strojwas MH, Cohen MS, et al. Patterns of brain activation in people at risk for Alzheimer's disease. N Engl J Med 2000;343:450-6.

25 Grady CL, Mclntosh AR, Beig S, et al. Evidence from functional neuroimaging of a compensatory prefrontal network in Alzheimer's disease. J Neurosci 2003:23:986-93.

26 DeKosky ST, Ikonomovic MD, Styren SD, et al. Upregulation of choline acetyltransferase activity in hippocampus and frontal cortex of elderly subjects with mild cognitive impairment. Ann Neurol 2002;51:145-55.

27 American Psychiatric Association. Diagnostic and statistical manual of mental disorders, 3rd ed, revised. Washington DC: American Psychiatric Association, 1987

28 McKhann G, Drachman D, Folstein M, et al. Clinical diagnosis of Alzheimer's disease: report of the NINCDS-ADRDA Work Group under the auspices of Department of Health and Human Services Task Force on Alzheimer's Disease. Neurology 1984;34:939-44.

29 Petersen RC, Stevens JC, Ganguli M, et al. Practical parameter: early detection of dementia: mild cognitive impairment (an evidence-based review) Report of the quality standards subcommittee of the American Academy of Neurology. Neurology 2001;56:1133-42.

30 Neary D, Snowden JS, Gustafson L, et al. Frontotemporal lobar degeneration a consensus on clinical diagnostic criteria. Neurology 1998;51:1546-54.
31 Folstein MF, Folstein SE, McHugh PR. "Mini-mental state". A practical method for grading the cognitive state of patients for the clinician. I Psychiatr Res 1975; 12:189-98.

32 Heaton RK. Wisconsin Card Sorting Test Manual. Odessa, Florida: Psychological Assessment Resources, 1981.

33 Seidman L, Pepple JR, Faraone SV, et al. Wisconsin Card Sorting Test over time in schizophrenia: preliminary evidence from clinical follow-up and neuroleptic reduction studies. Schizophr Res 1991;5:233-42.

34 Mazziotta JC, Toga AW, Evans A, et al. A probabilistic atlas of the human brain: theory and rationale for its development. The International Consortium for Brain Mapping (ICBM). Neuroimage 1995;2:89-101.

35 Bokde AL, Pietrini P, lbanez V, et al. The effect of brain atrophy on cerebral hypometabolism in the visual variant of Alzheimer disease. Arch Neurol 2001:58:480-6.

36 Ibanez V, Pietrini P, Alexander GE, et al. Regional glucose metabolic abnormalities are not the result of atrophy in Alzheimer's disease. Neurology 1998;50:1585-93.

37 Slansky I, Herholz K, Pietrzyk U, et al. Cognitive impairment in Alzheimer's disease correlates with ventricular width and atrophy-corrected cortical glucose metabolism. Neuroradiology 1995;37:270-7.

38 Friston KJ, Holmes AP, Worsley KJ, et al. Statistical parametric maps in functional imaging: a general linear approach. Hum Brain Mapping 1995;2:189-210

39 Bailey DL, Jones T, Friston KJ, et al. Physiological validation of statistical parametric mapping. J Cereb Blood Flow Metab 1991;11(Suppl 3):S150

40 Talairach J, Tournoux P. Co-planar stereotaxic atlas of the human brain 3-dimensional proportional system: an approach to cerebral imaging. Stuttgart: Georg Thieme Verlag, 1988.

41 Nagahama $\mathrm{Y}$, Fukuyama $\mathrm{H}$, Yamauchi $\mathrm{H}$, et al. Age-related changes in cerebral blood flow activation during a Card Sorting Test. Exp Brain Res 1997;114:571-7.

42 Costello CG. Research on symptoms versus research on syndromes. Arguments in favour of allocating more research time to the study of symptoms. Br J Psychiatry 1992;160:304-8.

43 Dolan RJ, Bench CJ, Liddle PF, et al. Dorsolateral prefrontal cortex dysfunction in the major psychoses; symptom or disease specificity? I Neurol Neurosurg Psychiatry 1993;56:1290-94.

44 Bradley KM, O'Sullivan VT, Soper NDW, et al. Cerebral perfusion SPET correlated with Braak pathological stage in Alzheimer's disease. Brain 2002; 125:1772-81

45 Nebu $A$, lkeda $M$, Fukuhara $R$, et al. Relationship between blood flow kinetics and severity of Alzheimer's disease: assessment of severity using a questionnaire-type examination, Alzheimer's disease assessment scale, cognitive sub-scale (ADAScog). Dement Geriatr Cogn Disord $2001 ; 12: 318-25$

46 Martin AJ, Friston KJ, Colebatch JG, et al. Decreases in regional cerebral blood flow with normal aging. J Cereb Blood Flow Metab 1991;1 1:684-9.

47 Mielke R, Kessler J, Szelies B, et al. Normal and pathological aging - findings of positron-emission-tomography. J Neural Transm 1998;105:821-37.

48 Matsuda H. Cerebral blood flow and metabolic abnormalities in Alzheimer's disease. Ann Nucl Med 2001;15:85-92.

49 Rogers RD, Andrews TC, Grasby PM, et al. Contrasting cortical and subcortical activations produced by attentional-set shiffing and reversal learning in humans. J Cogn Neurosci 2000;12:142-62.

50 Pollmann S, Weidner R, Muller HJ, et al. A fronto-posterior network involved in visual dimension changes. J Cogn Neurosci 2000;12:480-94.

51 Konishi S, Nakajima K, Uchida I, et al. Common inhibitory mechanism in human inferior prefrontal cortex revealed by event-related functional MRI. Brain 1999;122:981-91.

52 Menon V, Adleman NE, White CD, et al. Error-related brain activation during a Go/NoGo response inhibition task. Hum Brain Mapp 2001;12:131-43.

53 Rubia K, Russell T, Overmeyer S, et al. Mapping motor inhibition: conjunctive brain activations across different versions of go/no-go and stop tasks. Neuroimage $2001 ; 13: 250-61$.

54 Rushworth MF, Nixon PD, Eacott M, et al. Ventral prefrontal cortex is not essential for working memory. J Neurosci 1997:17:4829-38.

55 Iversen SD, Mishkin M. Perseverative interference in monkeys following selective lesions of the inferior prefrontal convexity. Exp Brain Res 1970;11:376-86

56 Dias R, Robbins TW, Roberts AC. Dissociation in prefrontal cortex of affective and attentional shifts. Nature 1996; 380:69-72.

57 Cools R, Clark L, Owen AM, et al. Defining the neural mechanisms of probablistic reversal learning using event-related functional magnetic resonance imaging. J Neurosci 2002;22:4563-7.

58 Petrides M. Impairments on nonspatial self-ordered and externally ordered working memory tasks after lesions of the mid-dorsal part of the lateral frontal cortex in the monkey. J Neurosci 1995; 15:359-75.

59 Owen AM, Evans AC, Petrides M. Evidence for a two-stage model of spatial working memory processing within the lateral frontal cortex: a positron emission tomography study. Cereb Cortex 1996;6:31-8.

60 Sandson J, Albert ML. Perseveration in behavioral neurology. Neurology 1987;37:1736-41

61 Fink GR, Dolan RJ, Halligan PW, et al. Space-based and object-based visual attention: shared and specific neural domains. Brain 1997;120:2013-28.

62 Wojciulik E, Kanwisher N. The generality of parietal involvement in visual attention. Neuron 1999;23:747-64.

63 Nagahama Y, Sadato N, Yamauchi $\mathrm{H}$, et al. Neural activity during attention shifts between object features. Neuroreport 1998;9:2633-8. 